\title{
NECESSARY CONDITIONS FOR FINITE CRITICAL SETS. MAPS WITH INFINITE CRITICAL SETS
}

\author{
Ioan Radu Peter - Cornel Pintea
}

\begin{abstract}
We provide necessary conditions on a given map, between two compact differential manifolds, for its critical set to be finite. As consequences of these conditions we also provide several examples of pairs of compact differential manifolds such that every map between them has infinite critical set.
\end{abstract}

\section{Introduction}

In the last decades the maps with small critical sets have been intensively studied by many authors, most of results concern information on the local behavior of maps themselves or even on their source and target manifolds. Andrica and Funar [1], [2] showed that the source compact $n$-dimensional manifold of a map with finitely many critical points is a connected sum of a finite covering of its target compact $n$-dimensional manifold and an exotic sphere. Later on, Funar [7] extended this type of results to higher codimension maps with finitely many critical points in which the role of finite covering maps is played by fibrations and the role of the exotic sphere is played by some homotopy sphere. Church and Timourian [3], [4] were able to control, in the small codimension cases (0, 1 and 2), the local behavior of a map with 0 -dimensional branch locus. Using a different approach, the second author [12], [13] showed, in the small

2010 Mathematics Subject Classification. Primary: 55Q05, 55N10.

Key words and phrases. Critical points; homotopy groups; low dimensional manifolds.

Cornel Pintea was supported by a grant of the Romanian National Authority for Scientific Research CNCS - UEFISCDI, project number PN-II-ID-PCE-2011-3-0024. 
codimension cases, that the homotopy groups of compact source and target manifolds of a map $f: M \longrightarrow N$ with finitely many critical points are, up to a certain rank, close to each other, as the fiber of the restriction

$$
M \backslash f^{-1}(B(f)) \stackrel{g}{\longrightarrow} N \backslash B(f), \quad p \mapsto f(p)
$$

is fully topologically controllable. We apply here this technique to obtain necessary conditions for finite critical sets in the higher codimension case. If, on the contrary, some homotopy groups of the two involved manifolds are 'away' from each other, then every map between the two manifolds has infinite critical set. This is the case for the examples we provide within the last section. The latter approach works in the higher codimension case as soon as we have some topological control on the fiber of restriction (1.1). We rely on the Poincaré conjecture, the Epstein classification theorem of three manifolds [5], [6], the Smale and Wall classification of 6-manifolds [16], [17] as well as on the Micallef-Moore theorem [11], for the topological control on the fiber of restriction (1.1).

A 3-manifold $X$ is said to be irreducible if every embedded two dimensional sphere bounds a 3-ball. A 3-manifold $X$ is called prime if $X$ cannot be written as a non-trivial connected sum of two manifolds, i.e. $X=X_{1} \sharp X_{2}$ implies that $X_{1}=S^{3}$ or $X_{2}=S^{3}$. Note that an irreducible 3-manifold is prime.

Recall that if $X$ is an orientable prime 3 -manifold with no spherical boundary components, then $X$ is either irreducible or $X=S^{1} \times S^{2}$ [9, Lemma 3.13]. In fact the product $X=S^{1} \times S^{2}$ is the only prime closed 3-manifold with infinite cyclic group.

Theorem 1.1 (Epstein [5], [6]). Let $X$ be a compact, orientable, irreducible 3-manifold with empty or toroidal boundary. If $\pi_{1}(X)$ is isomorphic to a direct product $G \times H$ of two non-trivial groups, then $X=S^{1} \times \Sigma$, with $\Sigma$ a surface.

Theorem 1.2 (Smale [16, Corollary 1.3]). The semi-group of 2-connected 6 -manifolds, whose operation is the connected sum $\sharp$, is generated by $S^{3} \times S^{3}$.

Theorem 1.3 (Wall [17]). Let $X$ be a closed, smooth, 1-connected 6-manifold. Then we can write $X$ as a connected sum $X_{1} \sharp X_{2}$, where $H_{3}\left(X_{1}\right)$ is finite and $X_{2}$ is a connected sum of copies of $S^{3} \times S^{3}$.

In other words, every simply connected 6 -manifold $X$ is diffeomorphic to a connected sum

$$
X \cong X_{1} \sharp\left(\sharp_{r}\left(S^{3} \times S^{3}\right)\right), \quad H_{3}\left(X_{1}, \mathbb{Z}\right)-\text { finite },
$$

and every 2-connected 6-manifold $Y$ is diffeomorphic to a connected sum

$$
Y \cong \sharp_{s}\left(S^{3} \times S^{3}\right) \text {. }
$$


TheOREM 1.4 (Micallef-Moore [11]). Let $M$ be a smooth, orientable, closed $n$-manifold such that $\pi_{1}(M)$ is a free group on $k$ generators and $\pi_{i}(M)=0$ for $2 \leq i \leq n / 2$. If $n \neq 4$ or $k=1$, then $M$ is homeomorphic to the connected sum of $k$ copies of $S^{1} \times S^{n-1}$.

Recall that the critical set of a differentiable map $f: M \rightarrow N$ is denoted by $C(f)$, its set of regular points $M \backslash C(f)$ by $R(f)$ and the set of its critical values $f(C(f))$ is denoted by $B(f)$. We rely on the restriction (1.1) of such a mapping $f: M \rightarrow N$, which is a fibration whenever $M$ and $N$ are compact connected manifolds such that $\operatorname{dim}(M) \geq \operatorname{dim}(N)$. The source and target manifolds of this restriction turn out to be connected under the countability assumption on the critical set $C(f)$. It therefore produces the following long homotopy sequence:

$(1.2) \rightarrow \pi_{r+1}(N \backslash B(f)) \longrightarrow \pi_{r}(F) \longrightarrow \pi_{r}\left(M \backslash f^{-1}(B(f))\right) \longrightarrow \pi_{r}(N \backslash B(f)) \rightarrow$

In fact we have

Proposition 1.5 ([13]). If $M^{n+k}, N^{n}$ are differential manifolds and $f: M \rightarrow$ $N$ is a smooth mapping with finite critical set, then

$$
\pi_{r}\left(M, M \backslash f^{-1}(B(f))\right) \cong 0 \cong \pi_{r}(N, N \backslash B(f)) \quad \text { for all } r \in\{1, \ldots, n-1\} .
$$

In particular, the open manifolds $M \backslash f^{-1}(B(f))$ and $N \backslash B(f)$ are connected and the inclusions $i: M \backslash f^{-1}(B(f)) \hookrightarrow M, j: N \backslash B(f) \hookrightarrow N$ are $(n-1)$-connected, i.e. the induced homotopy group homomorphisms

$$
\begin{aligned}
\pi_{k}(i): \pi_{k}\left(M \backslash f^{-1}(B(f))\right) & \rightarrow \pi_{k}(M), \\
\pi_{k}(j): \pi_{k}(N \backslash B(f)) & \rightarrow \pi_{k}(N)
\end{aligned}
$$

are isomorphisms for $k=1, \ldots, n-2$ and an epimorphism for $k=n-1$.

By using the long homotopy sequence (1.2) of the fibration

$$
F \stackrel{i_{F}}{\rightarrow} M \backslash f^{-1}(B(f)) \rightarrow N \backslash B(f)
$$

alongside isomorphisms (1.3) we get, for $r \leq n-3$, the picture

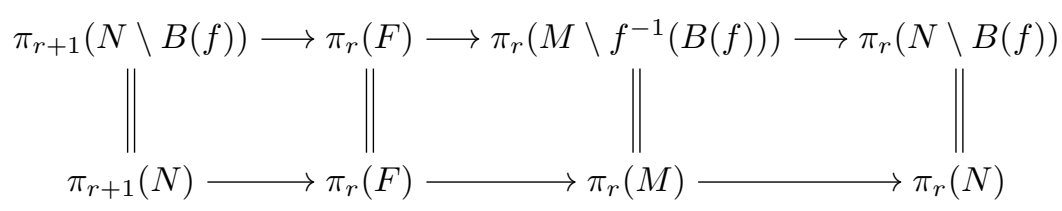

which produces, for $r \leq n-3$, the following finite exact sequence:

$$
\longrightarrow \pi_{r+1}(N) \longrightarrow \pi_{r}(F) \longrightarrow \pi_{r}(M) \longrightarrow \pi_{r}(N) \longrightarrow \pi_{r-1}(F) \longrightarrow
$$

The exact sequence (1.4) has some effective impact on the relation between the topologies of manifolds $M$ and $N$ only for $n \geq 4$, a good enough reason to work under this assumption all over the paper. 
We close this section by recalling a relation among ranks of terms of a finite exact sequence of finitely generated abelian groups, which will be repeatedly used along the paper in connection with the exact homotopy sequence of restriction (1.1). More precisely, if

$$
0 \longrightarrow G_{1} \longrightarrow \cdots \longrightarrow G_{n} \longrightarrow 0
$$

is an exact sequence of finitely generated abelian groups, then [14, p. 87]

$$
\sum_{i=1}^{n}(-1)^{i} \operatorname{rank}\left(G_{i}\right)=0 \text {. }
$$

\section{The main result}

Throughout this section $f: M \rightarrow N$ will be a map with finite critical set and $F$ the fiber of the restricted fibration (1.1), namely

$$
F \hookrightarrow M \backslash f^{-1}(B(f)) \stackrel{g}{\longrightarrow} N \backslash B(f), \quad p \mapsto f(p) .
$$

For the topological control of the fiber, we rely in this section on the Poincaré conjecture, the classification theorem of three manifolds [5], [6], the classification of the 6-manifolds [16], [17] as well as on the Micallef-Moore theorem [11].

TheOREM 2.1. Let $f: M^{n+k} \longrightarrow N^{n}$ be a map with finite critical set, where $M$ and $N$ are compact connected manifolds.

(a) If $k=3$, the induced group homomorphism $\pi_{2}(M) \rightarrow \pi_{2}(N)$ is trivial and the kernel $\operatorname{ker}\left[\pi_{1}(M) \rightarrow \pi_{1}(N)\right]$ is a subgroup of $\mathbb{Z}$, then $F=S^{3}$ or $F=S^{1} \times S^{2}$.

(b) If $k=3, M, N$ are orientable, $N$ is 3-connected, $\pi_{1}(M)$ is isomorphic to a direct product $G \times H$ of two nontrivial groups and $\pi_{2}(M) \equiv 0$, then $F=S^{1} \times \Sigma_{g}$, where $\Sigma_{g}$ is the compact orientable surface of some genus $g \geq 1$.

(c) If $k=6$ and the groups $\operatorname{ker}\left[\pi_{1}(M) \rightarrow \pi_{1}(N)\right], \pi_{2}(N)$ are trivial, then $F$ is diffeomorphic to some connected sum $X_{1} \sharp\left(\sharp_{r}\left(S^{3} \times S^{3}\right)\right)$. Under the stronger requirements on $M$ to be 3 -connected and $N$ to be $k$-connected for some $6 \leq k \leq n-4$, the diffeomorphism type of the fiber $F$ is a connected sum $\sharp_{s}\left(S^{3} \times S^{3}\right)$.

(d) If $k$ is even, $\pi_{1}(M)=\mathbb{Z}, \pi_{1}(N)$ is finite and $\pi_{i}(M)=\pi_{i}(N)=\pi_{k / 2+1}(N)$ $=0$ for all $2 \leq i \leq k / 2$, then $F$ is homeomorphic to $S^{1} \times S^{k-1}$.

Proof. Assume that the critical set $C(f)$ is finite for some $C^{1}$-smooth map $f: M \rightarrow N$.

(a) By using the exact homotopy sequence (1.4) corresponding to $r=1$, one obtains the exact sequence

$$
\cdots \longrightarrow \pi_{2}(M) \longrightarrow \pi_{2}(N) \longrightarrow \pi_{1}(F) \longrightarrow \pi_{1}(M) \longrightarrow \pi_{1}(N) .
$$


Since the induced group homomorphism $\pi_{2}(M) \rightarrow \pi_{2}(N)$ is trivial, it follows that the next group homomorphism is one to one, i.e. $\pi_{1}(F) \cong \operatorname{ker}\left[\pi_{1}(M) \rightarrow \pi_{1}(N)\right]$ is a subgroup of $\mathbb{Z}$. But the subgroups of $\mathbb{Z}$ are $m \mathbb{Z}, m \in \mathbb{Z}$, and the nontrivial ones are still isomorphic to $\mathbb{Z}$. Thus $\pi_{1}(F)$ is either trivial or $\pi_{1}(F) \cong \mathbb{Z}$, what shows that the fiber $F$ is either homeomorphic to $S^{3}$ or $F$ is homeomorphic to $S^{1} \times S^{2}$.

(b) The orientability of $M$ and $N$ insures, according with Hirsch [10, Lemma 4.1] adjusted to the exact sequence

$$
0 \longrightarrow \operatorname{ker}(d g) \longrightarrow T\left(M \backslash f^{-1}(B(f))\right) \longrightarrow T(N \backslash B(f)) \longrightarrow 0,
$$

the orientability of the integrable vector bundle $\operatorname{ker}(d g)$ and implicitly the orientability of its leaves which are the fibers of the restriction (1.1), namely

$$
M \backslash f^{-1}(B(f)) \stackrel{g}{\longrightarrow} N \backslash B(f), \quad p \mapsto f(p) .
$$

If $f: M \rightarrow N$ has finite critical set, then the fibration (1.1) produces the exact sequence (1.4), which, for $r \leq 2$, becomes

$\cdots \rightarrow \pi_{3}(N) \longrightarrow \pi_{2}(F) \longrightarrow \pi_{2}(M) \longrightarrow \pi_{2}(N) \longrightarrow \pi_{1}(F) \longrightarrow \pi_{1}(M) \longrightarrow \pi_{1}(N)$.

It shows that $\pi_{2}(F)$ is isomorphic to $\pi_{2}(M) \equiv 0$ and $\pi_{1}(F)$ is isomorphic to $\pi_{1}(M) \cong G \times H$. The triviality of $\pi_{2}(F)$ implies the irreducibility of $F$. By the Epstein theorem $[5,6]$ combined with our hypothesis, one can deduce that $F=S^{1} \times \Sigma_{g}$, where $\Sigma_{g}$ is the compact orientable surface of some genus $g \geq 1$.

(c) By using the exact homotopy sequence (1.4) corresponding to $r=1$, one obtains the following exact sequence:

$$
\cdots \rightarrow \pi_{2}(M) \longrightarrow \pi_{2}(N) \longrightarrow \pi_{1}(F) \longrightarrow \pi_{1}(M) \longrightarrow \pi_{1}(N) .
$$

Since the groups $\operatorname{ker}\left[\pi_{1}(M) \longrightarrow \pi_{1}(N)\right], \pi_{2}(N)$ are trivial, we obtain the simply connectedness of the fiber $F$, i.e. $\pi_{1}(F) \cong 0$. This shows that the closed 6 dimensional fiber $F$ of the restriction

$$
\left.f\right|_{M \backslash\left(f^{-1}(B(f))\right)}: M \backslash\left(f^{-1}(B(f))\right) \rightarrow N \backslash B(f)
$$

is, according to Wall's classification result, diffeomorphic to some connected sum $X_{1} \sharp\left(\sharp_{r}\left(S^{3} \times S^{3}\right)\right)$.

In the 3 -connected case of $M$ combined with the $k$-connectedness of $N$ we use the exact homotopy sequence (1.4) for $r \in\{1,2\}$. Taking into account the hypothesis, we obtain that $\pi_{1}(F) \cong 0 \cong \pi_{2}(F)$, i.e. $F$ is 2 -connected. Thus, the closed 6-dimensional fiber $F$ of the restriction

$$
\left.f\right|_{M \backslash\left(f^{-1}(B(f))\right)}: M \backslash\left(f^{-1}(B(f))\right) \rightarrow N \backslash B(f)
$$

is, according to Smale's classification result, diffeomorphic to some connected $\operatorname{sum} \sharp_{s}\left(S^{3} \times S^{3}\right)$. 
(d) The existence of a map $f: M \rightarrow N$ with finite critical set produces the exact sequence (1.4), which, for $2 \leq r \leq k / 2$, becomes

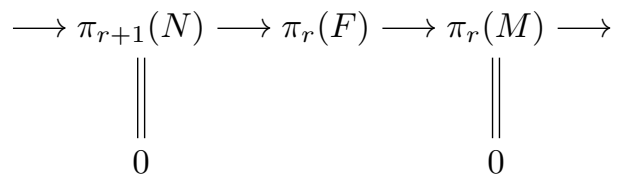

and this shows that $\pi_{j}(F)=0$ for $2 \leq i \leq k / 2$. The exact sequence (1.4) for $r=1$ becomes

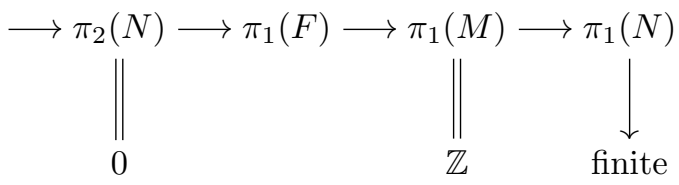

and this shows that $\pi_{1}(F)$ is isomorphic to one nontrivial subgroup of $\mathbb{Z}$, i.e. $\pi_{1}(F)$ is isomorphic to $\mathbb{Z}$. Therefore $F$ is homeomorphic, via the Micallef-Moore theorem, to $S^{1} \times S^{k-1}$.

COROLlary 2.2. Let $f: M^{n+k} \rightarrow N^{n}$ be a map with finite critical set, where $M$ and $N$ are compact connected manifolds.

(a) If $k=3, M, N$ are orientable, $N$ is 3-connected, $\pi_{1}(M)$ is isomorphic to a direct product $G \times H$ of two nontrivial groups and $\pi_{2}(M) \equiv 0$, then $\pi_{r}(M) \cong \pi_{r}(N)$ for every $3 \leq r \leq n-2$.

(b) If $k=6, M$ is 3-connected and $N$ is $k$-connected for some $6 \leq k \leq n-4$, then $H_{5}(M, \mathbb{Z}) \cong H_{5}(N, \mathbb{Z})$.

(c) If $k \geq 4$ is even, $\pi_{1}(M)=\mathbb{Z}, \pi_{1}(N)$ is finite and $\pi_{i}(M)=\pi_{i}(N)=$ $\pi_{k / 2+1}(N)=0$ for some $2 \leq i \leq k / 2$, then $\pi_{j}(M) \cong \pi_{j}(N)$ for every $k / 2<j<\min \{k-1, n-2\}$.

Proof. (a) According to Theorem 2.1 (a), the fiber is $F=S^{1} \times \Sigma_{g}$, where $\Sigma_{g}$ is the compact orientable surface of some genus $g \geq 1$. For $3 \leq r \leq n-2$, the exact sequence (1.4) becomes

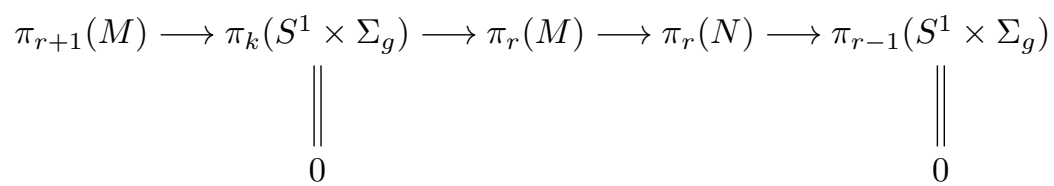

and this shows that $\pi_{r}(M) \cong \pi_{r}(N)$.

(b) The diffeomorphism type of the fiber $F$ is, according to Theorem 2.1 (c), a connected sum $\sharp_{s}\left(S^{3} \times S^{3}\right)$. The $k$-connectedness of $N \backslash B(f)$ combined with the 2 -connectedness of the fiber allow us to use the Serre exact homology sequence associated to the fibration

$$
\sharp_{s}\left(S^{3} \times S^{3}\right) \hookrightarrow M \backslash\left(f^{-1}(B(f))\right) \rightarrow N \backslash B(f) .
$$


Taking into account that

$$
H_{i}\left(M \backslash\left(f^{-1}(B(f))\right), \mathbb{Z}\right) \cong H_{i}(M, \mathbb{Z}), \quad H_{i}(N \backslash B(f), \mathbb{Z}) \cong H_{i}(N, \mathbb{Z}),
$$

for $i \leq n-2$, the Serre exact homology sequence with integer coefficients associated to the fibration $(2.1)$ is

$$
H_{r+2}\left(\sharp_{s}\left(S^{3} \times S^{3}\right)\right) \longrightarrow H_{r+2}(M) \longrightarrow H_{r+2}(N) \longrightarrow H_{r+1}\left(\sharp_{s}\left(S^{3} \times S^{3}\right)\right) \rightarrow \cdots
$$

We particularly obtain

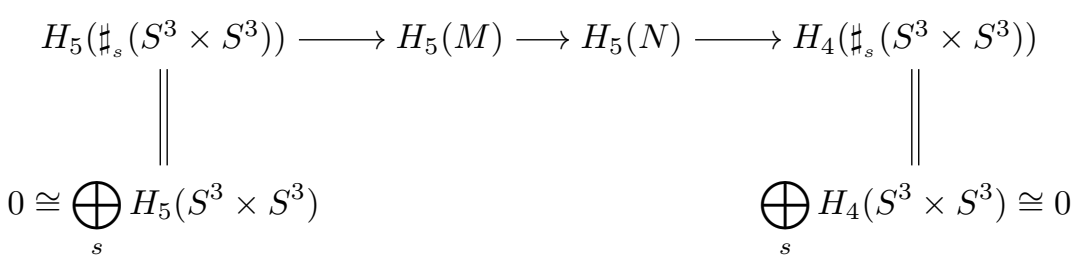

which shows that $H_{5}(M, \mathbb{Z}) \cong H_{5}(N, \mathbb{Z})$.

(c) The diffeomorphism type of the fiber $F$ is, according to Theorem $2.1(2.1)$, $S^{1} \times S^{k-1}$. By using the sequence (1.4) for some $\frac{k}{2}<r \leq n-2$, one obtains the following picture:

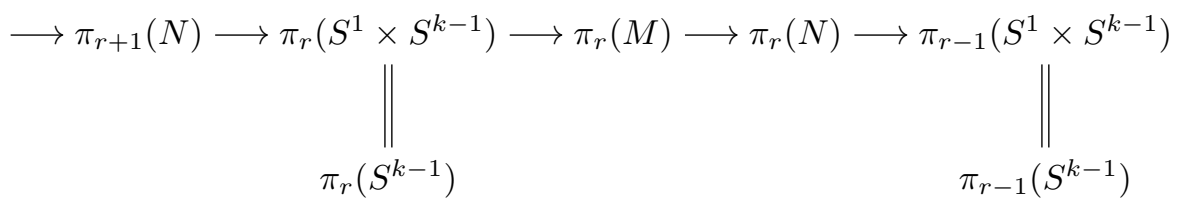

which leads us to the following exact sequences:

$$
\begin{aligned}
\rightarrow \pi_{r+1}(N) \longrightarrow \pi_{r}\left(S^{k-1}\right) \longrightarrow & \pi_{r}(M) \longrightarrow \\
& \longrightarrow \pi_{r}(N) \longrightarrow \pi_{r-1}\left(S^{k-1}\right) \longrightarrow \pi_{r-1}(N) .
\end{aligned}
$$

If $k / 2<j<\min \{k-1, n-2\}$, then $\pi_{j}\left(S^{k-1}\right)=0=\pi_{j-1}\left(S^{k-1}\right)$ and the sequence (2.2) shows, for $r=j$, that $\pi_{j}(M) \cong \pi_{j}(N)$.

While Corollary 2.2 used the full topological control on the fiber of the restriction (1.1) and the exactness of sequences (1.4) for higher ranks, the next corollary is going to exploit relation (1.5) in relation with the exact sequence (1.4).

COROllary 2.3. Let $f: M^{n+k} \longrightarrow N^{n}$ be a map with finite critical set, where $M$ and $N$ are compact connected manifolds with finitely generated homotopy groups.

(a) If $k=3, \pi_{1}(M), \pi_{1}(N)$ are abelian groups, the induced group homomorphism $\pi_{2}(M) \rightarrow \pi_{2}(N)$ is trivial and the kernel $\operatorname{ker}\left[\pi_{1}(M) \rightarrow \pi_{1}(N)\right]$ is a subgroup of $\mathbb{Z}$, then $\operatorname{rank}\left(\pi_{r}(M)\right) \leq \operatorname{rank}\left(\pi_{r}(N)\right)+1$, for all $1 \leq r \leq$ $n-2$. 
(b) If $k=3, M, N$ are orientable, $N$ is 3-connected, $\pi_{1}(M)$ is isomorphic to a direct product $G \times H$ of two nontrivial groups and $\pi_{2}(M) \equiv 0$, then $\operatorname{rank}\left(\pi_{2}(M)\right) \leq \operatorname{rank}\left(\pi_{2}(N)\right)$.

(c) If $k$ is even, $\pi_{1}(M)=\mathbb{Z}, \pi_{1}(N)$ is finite and

$$
\pi_{i}(M)=\pi_{i}(N)=\pi_{k / 2+1}(N)=0, \quad \text { for all } 2 \leq i \leq \frac{k}{2},
$$

then $\operatorname{rank}\left(\pi_{j}(M)\right) \leq \operatorname{rank}\left(\pi_{j}(N)\right)+2$ for every $k-1 \leq j \leq n-2$.

Proof. (a) According to Theorem 2.1 (a), the fiber is $F=S^{3}$ or $F=S^{1} \times S^{2}$ and therefore $\operatorname{rank}\left(\pi_{1}(F)\right) \leq 1$. The exact sequence (1.4) produces the following short exact sequences:

$$
\begin{aligned}
0 & \longrightarrow \pi_{1}(F) \longrightarrow \pi_{1}(M) \longrightarrow G \longrightarrow 0 \\
0 \longrightarrow H & \longrightarrow \pi_{r}(M) \longrightarrow \pi_{r}(N) \longrightarrow K \longrightarrow 0,
\end{aligned}
$$

where

$$
\begin{aligned}
& G=\operatorname{range}\left(\pi_{1}(M) \rightarrow \pi_{1}(N)\right), \\
& H=\operatorname{range}\left(\pi_{r}(F) \rightarrow \pi_{r}(M)\right), \\
& K=\operatorname{range}\left(\pi_{r}(N) \rightarrow \pi_{r-1}(F)\right) .
\end{aligned}
$$

Thus, the ranks of groups involved in the exact sequence (2.3) are related by

$$
\operatorname{rank}\left(\pi_{1}(M)\right)=\operatorname{rank}\left(\pi_{1}(F)\right)+\operatorname{rank}(G) \leq 1+\operatorname{rank}\left(\pi_{1}(M)\right) .
$$

Similarly, the ranks of the groups involved in the exact sequence (2.4) are related by $\operatorname{rank}(H)-\operatorname{rank}\left(\pi_{r}(M)\right)+\operatorname{rank}\left(\pi_{r}(N)\right)-\operatorname{rank}(K)=0$, what implies

$$
\begin{aligned}
\operatorname{rank}\left(\pi_{r}(M)\right) & =\operatorname{rank}(H)+\operatorname{rank}\left(\pi_{r}(N)\right)-\operatorname{rank}(K) \\
& \leq \operatorname{rank}(H)+\operatorname{rank}\left(\pi_{r}(N)\right) \\
& \leq \operatorname{rank}\left(\pi_{r}(F)\right)+\operatorname{rank}\left(\pi_{r}(N)\right) \leq \operatorname{rank}\left(\pi_{r}(N)\right)+1 .
\end{aligned}
$$

(b) According to Theorem 2.1 (b), the fiber is $F=S^{1} \times \Sigma_{g}$, where $\Sigma_{g}$ is the compact orientable surface of some genus $g \geq 1$. The exact sequence (1.4) for $k=2$ becomes

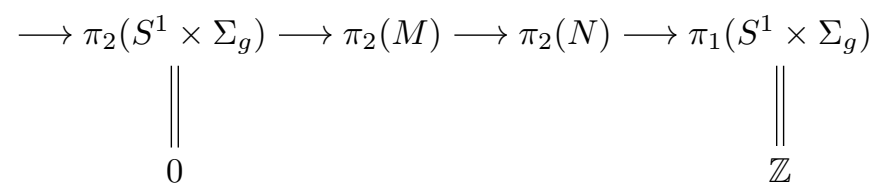

and this shows that $\pi_{2}(M)$ is a subgroup of $\pi_{2}(N)$.

(c) According to Theorem 2.1 (d), the fiber is $F=S^{1} \times S^{k-1}$. Assume that $k-1 \leq r \leq n-2$. From the exact sequence (2.2) we may single out the following short exact sequence:

$$
0 \longrightarrow G_{r} \longrightarrow \pi_{r}\left(S^{k-1}\right) \longrightarrow \pi_{r}(M) \longrightarrow \pi_{r}(N) \longrightarrow \pi_{r-1}\left(S^{k-1}\right) \longrightarrow H_{r} \longrightarrow 0
$$


where $G_{r}=\operatorname{range}\left(\pi_{r+1}(N) \rightarrow \pi_{r}\left(S^{k-1}\right)\right), H_{r}=\operatorname{range}\left(\pi_{r-1}\left(S^{k-1}\right) \rightarrow \pi_{r-1}(N)\right)$. Thus, the ranks of groups involved in the exact sequence (2.5) are related by

$$
\begin{aligned}
\operatorname{rank}\left(G_{r}\right)-\operatorname{rank}\left(\pi_{r}\left(S^{k-1}\right)\right)+\operatorname{rank}( & \left.\pi_{r}(M)\right)-\operatorname{rank}\left(\pi_{r}(N)\right) \\
& +\operatorname{rank}\left(\pi_{r-1}\left(S^{k-1}\right)\right)-\operatorname{rank}\left(H_{r}\right)=0,
\end{aligned}
$$

what shows that

$$
\begin{aligned}
& \operatorname{rank}\left(\pi_{r}(M)\right)-\operatorname{rank}\left(\pi_{r}(N)\right) \\
& \quad=\operatorname{rank}\left(\pi_{r}\left(S^{k-1}\right)\right)-\operatorname{rank}\left(\pi_{r-1}\left(S^{k-1}\right)\right)+\operatorname{rank}\left(H_{r}\right)-\operatorname{rank}\left(G_{r}\right) \\
& \leq 1+\operatorname{rank}\left(H_{r}\right) \leq 2 .
\end{aligned}
$$

The inequality $\operatorname{rank}\left(\pi_{r}\left(S^{k-1}\right)\right)-\operatorname{rank}\left(\pi_{r-1}\left(S^{k-1}\right)\right)-\operatorname{rank}\left(G_{r}\right) \leq 1$ follows from the finiteness of all homotopy groups of spheres, except for those of the form $\pi_{j}\left(S^{j}\right)$ or $\pi_{4 j-1}\left(S^{2 j}\right)$, when the group is the product of the infinite cyclic group with a finite abelian group [15].

\section{Examples of maps with infinite critical sets}

If one conclusion of a certain statement of Corollaries 2.2 or 2.3 is violated by some pair $(M, N)$ of manifolds, then every map $f: M \rightarrow N$ has infinite critical set if the pair is subject to the hypotheses of that statement. The potential of this observation to produce pairs of manifolds $(M, N)$ with infinite critical sets for all maps $f: M \longrightarrow N$ is rather reach and we shall present here a few of them. Homotopy and homology groups of the source and target manifolds are used and we refer the reader to [8] for their computation.

EXAMPLE 3.1. If $n \geq 8$ and $g \geq 1$, then every $C^{1}$-smooth map

$$
f: S^{3} \times S^{1} \times \Sigma_{g} \times S^{n-3} \rightarrow S^{4} \times S^{n-4}
$$

has, according to Corollary 2.2 (a), infinite critical set, where $\Sigma_{g}$ stands for the compact orientable surface of genus $g$. Indeed $\pi_{1}\left(S^{3} \times S^{1} \times \Sigma_{g} \times S^{n-3}\right)$ is the product $\mathbb{Z} \times \pi_{1}\left(\Sigma_{g}\right), \pi_{2}\left(S^{3} \times S^{1} \times \Sigma_{g} \times S^{n-3}\right)=0$ and

$$
\pi_{4}\left(S^{3} \times S^{1} \times \Sigma_{g} \times S^{n-3}\right) \cong 0 \nsucceq \pi_{4}\left(S^{4} \times S^{n-4}\right) .
$$

Let us denote by $\Sigma_{g}^{q}$ the product $\Sigma_{g} \times \ldots \times \Sigma_{g}$ of $q$ copies of $\Sigma_{g}$.

EXAmPle 3.2. Every $C^{1}$-smooth map $f: M \longrightarrow N$ has infinite critical set for the following pairs of manifolds:

(a) $M=S^{1} \times\left(S^{2}\right)^{m+1}$ and $N=S^{2 m}, m \geq 2$.

(b) $M=S^{1} \times S^{2} \times\left(S^{4}\right)^{n}$ and $N=\Sigma_{g}^{2 n}, n \geq 2$.

(c) $M=S^{1} \times \ldots \times S^{4 k}$ and $N=\Sigma_{g}^{(k+1)(4 k-3)}, k \geq 1$.

(d) $M=S^{1} \times S^{2} \times \mathbb{R} \mathbb{P}^{p}$ and $N=S^{p}, p \geq 4$. 
Indeed, the first three items follow from Corollary $2.3(\mathrm{a})$, as $\pi_{2}\left(S^{1} \times\left(S^{2}\right)^{m+1}\right)=$ $\mathbb{Z}^{m+1}, \pi_{4}\left(S^{1} \times S^{2} \times\left(S^{4}\right)^{n}\right)=\mathbb{Z}_{2} \times \mathbb{Z}^{n}$ and $\mathbb{Z} \times \mathbb{Z}$ is a subgroup of the third homotopy group $\pi_{3}\left(S^{1} \times \ldots \times S^{4 k}\right)$. The last item follows from Corollary 2.3 (b), as $\pi_{1}\left(S^{1} \times S^{2} \times \mathbb{R} \mathbb{P}^{p}\right)=\mathbb{Z} \times \mathbb{Z}_{2}$ and $\pi_{2}\left(S^{1} \times S^{2} \times \mathbb{R} \mathbb{P}^{p}\right)=\mathbb{Z}$.

EXAMPLE 3.3 .

(a) Every $C^{1}$-smooth map $f$ : $\sharp_{r}\left(S^{2} \times S^{3} \times S^{n+1}\right) \rightarrow \sharp_{s}\left(S^{p} \times S^{n-p}\right)$ has infinite critical set if $p, n-p \geq 5$ and $r, s \geq 1$.

(b) Every differentiable map $f: \sharp_{r}\left(S^{5} \times S^{n+1}\right) \rightarrow \sharp_{s}\left(S^{6} \times S^{n-6}\right)$ has infinite critical set if $n \geq 12$ and $r, s \geq 1$.

Since each of the homology groups $H_{5}\left(\sharp_{r}\left(S^{2} \times S^{3} \times S^{n+1}\right), \mathbb{Z}\right), H_{5}\left(\sharp_{r}\left(S^{5} \times S^{n+1}\right), \mathbb{Z}\right)$ has an infinite cyclic subgroup and $H_{5}\left(\sharp_{s}\left(S^{p} \times S^{n-p}\right), \mathbb{Z}\right) \cong 0 \cong H_{5}\left(\sharp_{s}\left(S^{6} \times\right.\right.$ $\left.\left.S^{n-6}\right), \mathbb{Z}\right)$, the two items follow via Corollary $2.2(\mathrm{~b})$.

EXAMPLE 3.4.

(a) If $k \geq 2$ and $n \geq \min \{5, k+3\}$, then every $C^{1}$-smooth map $f: S^{1} \times$ $S^{n+k-2} \times S^{k+1} \rightarrow \mathbb{R P}^{n}$ has infinite critical set.

(b) If and $k \geq 2$ and $n \geq k+4$ then every $C^{1}$-smooth map

$$
g: S^{1} \times S^{n-3} \times S^{k+1} \times S^{k+1} \rightarrow \mathbb{R P}^{n}
$$

has infinite critical set.

The two items follow via Corollary 2.2 (c). Indeed,

$$
\pi_{1}\left(S^{1} \times S^{n+k-2} \times S^{k+1}\right) \cong \mathbb{Z} \cong \pi_{1}\left(S^{1} \times S^{n-3} \times S^{k+1} \times S^{k+1}\right)
$$

and $\pi_{1}\left(\mathbb{R P}^{n}\right) \cong \mathbb{Z}_{2}$. On the other hand, for $2 \leq i \leq k$, one has

$$
\pi_{i}\left(S^{1} \times S^{n+k-2} \times S^{k+1}\right) \cong 0 \cong \pi_{i}\left(S^{1} \times S^{n-3} \times S^{k+1} \times S^{k+1}\right)
$$

and $\pi_{j}\left(\mathbb{R} \mathbb{P}^{n}\right) \cong 0$ for all $2 \leq j \leq n-2$. Finally, $\pi_{k+1}\left(\mathbb{R} \mathbb{P}^{n}\right) \cong 0$ and

$$
\pi_{k+1}\left(S^{1} \times S^{n+k-2} \times S^{k+1}\right), \quad \pi_{k+1}\left(S^{1} \times S^{n-3} \times S^{k+1} \times S^{k+1}\right) \not 0,
$$

as the first $(k+1)$-homotopy group has an infinite cyclic subgroup and the second $(k+1)$-homotopy group has a free abelian subgroup with two generators.

Acknowledgements. The authors are grateful to the anonymous referee for the careful reading of the manuscript and the valuable suggestions which lead to the final improved presentation.

\section{REFERENCES}

[1] D. Andrica And L. Funar, On smooth maps with finitely many critical points, J. London Math. Soc. (2) 69 (2004), 783-800.

[2] D. ANDrica AND L. Funar, On smooth maps with finitely many critical points. Addendum, J. London Math. Soc. (2) 73 (2006), 231-236.

[3] P.T. Church and J .G. Timourian, Differentiable maps with 0-dimensional critical set I, Pacific J. Math. 41, No. 3 (1972), 615-630. 
[4] - Maps with 0-dimensional critical set, Pacific J. Math. 57, No. 1 (1975), 59-66.

[5] D.B.A. Epstein, Factorization of 3-manifolds, Comment. Math. Helv. 36 (1961), 91-102.

[6] _ Ends, Topology of 3-manifolds and related topics (Proc. The Univ. of Georgia Institute, 1961), Prentice-Hall, Englewood Cliffs, N.J., 1962, 110-117.

[7] L. FunAR, Global classification of isolated singularities in dimensions $(4,3)$ and $(8,5)$, Ann. Scuola Norm. Sup. Pisa Cl. Sci. (5) Vol. X (2011), 819-861.

[8] A. Hatcher, Algebraic Topology, Cambridge University Press, 2002.

[9] J. Hempel, 3-Manifolds, Ann. of Math. Studies, no. 86. Princeton University Press, Princeton, N. J., 1976.

[10] M.W. HiRsch, Differential Topology, Springer, 1976.

[11] M.J. Micallef AND J.D. Moore, Minimal two-spheres and the topology of manifolds with positive curvature on totally isotropic two-planes, Ann. of Math. (2) 127 (1988), no. 1, 199-227.

[12] C. PinteA, Differentiable mappings with an infinite number of critical points, Proc. Amer. Math. Soc., Vol. 128, No. 11. (2000), 3435-3444.

[13] _ A measure of the deviation from there being fibrations between a pair of compact manifolds, Diff. Geom. Appl. 24 (2006), 579-587.

[14] J.J. Rotman, An Introduction to Algebraic Topology, Springer Verlag, 1988.

[15] J.-P. SERre, Homologie singulire des espaces fibrs. Applications, Ann. Math. 54 (3) (1951), 425-505.

[16] S. Smale, On the structure of manifolds, Amer. J. Math. 84 (1962), 387-399.

[17] C.T.C. WALl, Classification problems in differential topology. V. On certain 6-manifolds, Invent. Math. 1 (1966), 355-374.

Manuscript received November 9, 2014

accepted November 29, 2015

IOAN RADU PETER

Department of Mathematics

Technical University of Cluj-Napoca

Str. Memorandumului nr. 28

400114, Cluj-Napoca, ROMANIA

E-mail address: ioan.radu.peter@math.utcluj.ro

Cornel Pintea

Department of Mathematics

"Babeş-Bolyai" University, Cluj-Napoca

M. Kogălniceanu 1

Cluj-Napoca, ROMANIA

E-mail address: cpintea@math.ubbcluj.ro 